\title{
Survival probability of a particle in a sea of mobile traps: A tale of tails
}

\author{
S.B. Yuste ${ }^{1}$, G.Oshanin ${ }^{2}$, K.Lindenberg ${ }^{3}$, O.Bénichou ${ }^{2}$ and J.Klafter ${ }^{4}$ \\ ${ }^{1}$ Departamento de Física, Universidad de Extremadura, E-06071 Badajoz, Spain \\ ${ }^{2}$ Laboratoire de Physique Théorique de la Matière Condensée, \\ Université Pierre et Marie Curie, 4 Place Jussieu, 75252 Paris, France \\ ${ }^{3}$ Department of Chemistry and Biochemistry, University of California, San Diego, La Jolla, CA 92093-0340 USA \\ ${ }^{4}$ School of Chemistry, Tel Aviv University, 69978 Tel Aviv, Israel
}

(Dated: October 28, 2018)

\begin{abstract}
We study the long-time tails of the survival probability $P(t)$ of an $A$ particle diffusing in $d$-dimensional media in the presence of a concentration $\rho$ of traps $B$ that move sub-diffusively, such that the mean square displacement of each trap grows as $t^{\gamma}$ with $0 \leq \gamma \leq 1$. Starting from a continuous time random walk (CTRW) description of the motion of the particle and of the traps, we derive lower and upper bounds for $P(t)$ and show that for $\gamma \leq 2 /(d+2)$ these bounds coincide asymptotically, thus determining asymptotically exact results. The asymptotic decay law in this regime is exactly that obtained for immobile traps. This means that for sufficiently subdiffusive traps, the moving $A$ particle sees the traps as essentially immobile, and Lifshitz or trapping tails remain unchanged. For $\gamma>2 /(d+2)$ and $d \leq 2$ the upper and lower bounds again coincide, leading to a decay law equal to that of a stationary particle. Thus, in this regime the moving traps see the particle as essentially immobile. For $d>2$, however, the upper and lower bounds in this $\gamma$ regime no longer coincide and the decay law for the survival probability of the $A$ particle remains ambiguous.
\end{abstract}

PACS numbers: 02.50.-r,82.40.-g,89.75.Da

\section{INTRODUCTION}

In the early 1960's, I.M. Lifshitz published his famous analysis of the low-energy tails of the density $N(E)$ of states of an electron in a medium with randomly scattered immobile impurities [1, 2]. He demonstrated that, in $d$-dimensions, the spectrum $N(E)$ of the random Schrödinger operator decays as

$$
N(E) \sim \exp \left(- \text { const } E^{-d / 2}\right), \quad E \rightarrow 0 .
$$

This exponential decay is in striking contrast to the polynomial decay associated with a periodic Schrödinger operator, and is caused by the presence of arbitrarily large, albeit rare, regions without impurities.

A decade later Balagurov and Vaks [3] and Donsker and Varadhan [4] published their celebrated work on trapping kinetics in a medium with randomly placed immobile traps (called $B$ ). They found that the long-time decay of the survival probability $P^{B}(t)$ of a particle (called $A$ ) diffusing in such a $d$ dimensional medium follows the stretched-exponential law

$$
P^{B}(t) \sim \exp \left(-k_{d} \rho^{2 /(d+2)}\left(D_{A} t\right)^{d /(d+2)}\right),
$$

where $\rho$ is the mean density of traps, $D_{A}$ is the particle diffusion coefficient, and $k_{d}$ is the $d$-dependent constant [4]

$$
k_{d}=\left(\frac{d+2}{2 d}\right)\left(d v_{d}\right)^{2 /(d+2)}\left(2 z_{d}^{2}\right)^{d /(d+2)} .
$$

Here $z_{d}$ is the first zero of the Bessel function $J_{(d-2) / 2}(z)$ and $v_{d}=2 \pi^{d / 2} /[d \Gamma(d / 2)]$ denotes the volume of a $d$-dimensional sphere of unit radius. The superscript $B$ on the survival probability emphasizes that the traps $B$ are immobile. Balagurov and Vaks obtained the decay form in Eq. (2) exactly for $d=1$. While they furthermore deduced this behavior for general $d$ by noticing the close mathematical connection between the trapping problem and the Lifshitz problem, Donsker and Varadhan were the first to obtain the rigorous exact solution of this essentially many-body problem in $d$ dimensions. They determined not only the time dependence but also the decay coefficient $k_{d}$ of Eq. (3). Note that this law stands in stark contrast to the purely exponential decay predicted by standard chemical kinetics for the reaction $A+B \rightarrow B$ of mobile particles and traps, and even to the predictions of the Smoluchowski approach based on a reaction-diffusion equation,

$$
P(t) \sim \begin{cases}\exp \left[-\rho(4 D t / \pi)^{1 / 2}\right], & d=1 \\ \exp \left[-4 \pi \rho D t / \ln \left(D t / a^{2}\right)\right], & d=2 \\ \exp (-4 \pi \rho D t), & d=3\end{cases}
$$

where $D$ is the sum of the diffusion coefficients of the particle and the traps [5]. 
In mathematical analogy with the source of the Lifshitz tail, the decay law (2) arises from the presence of arbitrarily large regions without traps in which the particle can diffuse for a relatively long time before being trapped. Although the survival probability of a particle in any one such region is purely exponential, the ensemble average over a random distribution of such trap-free voids produces the anomalously slow decay.

The decay law in Eq. (2) has been also generalized to trapping reactions on fractals and in inhomogeneous structures [6], to $A$ particles performing subdiffusive motion [7, 8, 9, 10] or attached to the extremities of polymer chains [11], and also to agglomerated random distributions of traps distributed on immobile polymer chains in solution [12] or in clusters [13]. The history of this problem and many other results have been summarized in several reviews (see, e.g., [14, 15]).

The survival probability Eq. (2) [as well as the Lifshitz tail result of Eq. (11)] is valid only when the traps (or impurities) are strictly immobile. Indeed, if one allows them to diffuse, no matter how small the diffusion coefficient, the particle survival probability is described by a faster decay law. As proved by Bramson and Lebowitz [16] (see also [17]), when both species diffuse, the survival probability of the $A$ particle at long times is given by

$$
P(t) \sim \begin{cases}\exp \left(-\rho c_{1} t^{1 / 2}\right), & d=1 \\ \exp \left(-\rho c_{2} t / \ln t\right), & d=2 \\ \exp \left(-\rho c_{3} t\right), & d=3 .\end{cases}
$$

The time dependences are the same as in the Smoluchowski problem, but the constants $c_{1}, c_{2}$ and $c_{3}$ are in general different, may depend on the diffusion coefficients of the particle and of the traps (but see below), and were not determined in this original work.

When traps diffuse, the many-body trapping effects captured by Eq. (2) are thus no longer applicable and the $A$ particle survival probability decays with time according to the faster time dependencies in Eq. (5). The underlying fluctuation mechanism governing the trapping dynamics has changed when the traps are allowed to move. While the time dependence in the decay laws (5) is consistent with Smoluchowski-like results, which in fact represent a two-body approximation, the decay amplitudes $c_{d}$ are not simply functions of the sum of the diffusion coefficients. In particular, the exact form (including the coefficients) of the leading large- $t$ behavior for $d=1$ and $d=2$ has only recently been found by Bray and Blythe [18] and, surprisingly, $c_{1}$ and $c_{2}$ depend only on the diffusion coefficient $D_{B}$ of the traps and are independent of $D_{A}$, the diffusion coefficient of the $A$ particle! This implies that the survival probability of the diffusing $A$ particle in this scenario is asymptotically identical to that of an $A$ particle that remains still, that is, to $P^{A}(t)$.

This remarkable result has subsequently been extended to systems in which both the particles and the traps move subdiffusively [7, 8, 9, 10]. In particular, it is again found that the survival probability of a subdiffusive $A$ particle in a sea of subdiffusive traps is identical to that of and $A$ particle that remains still (as in the Bramson-Lebowitz scenario), $P(t) \sim P^{A}(t)$. However, it is still not clear what happens when traps move subdiffusively and the particle is diffusive. In one dimension it was shown in [7, 8] that the asymptotic survival probability of the diffusing particle is the same when surrounded by subdiffusive traps characterized by a mean square displacement that grows as $t^{\gamma}$ with $\gamma \leq 1$ as it is for a stationary particle, provided the subdiffusive traps move sufficiently rapildy $(\gamma>2 / 3)$, again akin to the Bramson-Lebowitz scenario. However, few results seem to be currently available for $d>1$ and/or if the subdiffusive traps are "too slow." An interesting question is then the following: will the trapping tails associated with immobile traps withstand sufficiently slow sub-diffusion, thus leading to a survival probability such as that of the Donsker-Varadhan result, or will they switch to appropriately rescaled Bramson-Lebowitz forms? This is the main question that we pose in this paper.

In this pursuit, we follow the general idea [18] of constructing lower and upper bounds on the survival probability of a diffusive particle in a sea of subdiffusive traps whose motion is described by a continuous time random walk that starts at time $t=0$ [19]. If these bounds coincide asymptotically, then we can extract an exact asymptotic result for the survival probability of the particle. We write the asymptotic survival probability in the form

$$
P(t) \sim \begin{cases}\exp \left(-\theta t^{z}\right), & d \neq 2 \\ \exp \left(-\theta t^{z} / \ln t\right) & d=2\end{cases}
$$

where the constants $\theta$ and $z$ depend on $\gamma$ and on dimension, and explore whether a convergence of upper and lower bounds provides the exponents $z$ and perhaps even the exponential prefactor $\theta$. We broadly anticipate our results by noting that if the subdiffusive particles are sufficiently slow, specifically if $\gamma \leq 2 /(d+2)$, these bounds lead to a Donsker-Varadhan behavior, and the Lifshitz or trapping tails thus remain unchanged. If $\gamma>2 /(d+2)$ and $d \leq 2$, then the bounds lead to a Bramson-Lebowitz behavior that is, a survival probability behavior associated with a stationary particle (note that, albeit for a different system, this result is implicit in [10]; that work addresses diffusive particles in a fractal medium, while here we are considering subdiffusive motion in Euclidean geometries). However, if $\gamma>2 /(d+2)$ and $d>2$ we are not able to establish a unique asymptotic behavior. The detailed results are exhibited later in the paper.

In Sec. [I] we formulate the model of moving particle and traps. In Sec. [II] we calculate a lower bound on the survival probability of the particle, and in Sec. IV we obtain two upper bounds. The consequences of these bounds on the survival probability are collected in Sec. $\mathrm{V}$. A brief recapitulation of the results is given in the concluding section. 


\section{THE MODEL}

In this section we formulate the general model that allows us to highlight the approximations made to obtain upper and lower bounds on the survival probability. Consider a $d$-dimensional system of volume $V$ containing a single diffusive $A$ particle of radius $a$ and $K$ randomly moving point traps $B$ (a finite trap radius would add nothing interesting to the problem for our purposes). The initial position of $A$ is the origin, and one realization of its trajectory is denoted by $\boldsymbol{a}_{t}$. The starting points $B_{0}^{(k)}, k=1,2, \cdots K$ of the traps are randomly (Poisson) distributed throughout the volume, and a trajectory of the $k$ th trap is denoted by $\boldsymbol{B}_{t}^{(k)}=\boldsymbol{B}_{0}^{(k)}+\boldsymbol{b}_{t}^{(k)}$.

Next we define

$$
v(\boldsymbol{x})= \begin{cases}\infty, & |\boldsymbol{x}| \leq a \\ 0, & \text { otherwise }\end{cases}
$$

The indicator function $\Psi\left[\boldsymbol{a}_{t},\left\{\boldsymbol{b}_{t}^{(k)}\right\}\right]$ of the event that the $A$ particle has not met any of the $B$ s up to time $t$ for a given realization of their trajectories can then be written as

$$
\Psi\left[\boldsymbol{a}_{t},\left\{\boldsymbol{b}_{t}^{(k)}\right\}\right]=\prod_{k=1}^{K} \Psi\left[\boldsymbol{a}_{t}, \boldsymbol{b}_{t}^{(k)}\right]=\prod_{k=1}^{K} \exp \left[-\int_{0}^{t} v\left(\boldsymbol{a}_{\tau}-\boldsymbol{b}_{\tau}^{(k)}-\boldsymbol{B}_{0}^{(k)}\right) d \tau\right] .
$$

Consequently the $A$ particle survival probability is

$$
P(t)=E^{(a)}\left\{\prod_{k=1}^{K}\left(\frac{1}{V} \int_{V} d \boldsymbol{B}_{0}^{(k)} E^{(b)}\left\{\Psi\left[\boldsymbol{a}_{t}, \boldsymbol{b}_{t}^{(k)}\right]\right\}\right)\right\}
$$

where the symbol $E^{(a)}\{\ldots\}$ denotes an average over all $A$ particle trajectories $\boldsymbol{a}_{t}$ that start at the origin. The symbol $E^{(b)}\{\ldots\}$ denotes an average over all $B$ trajectories $\boldsymbol{b}_{t}$ whose starting point is $B_{0}$. We have labeled each of the latter trajectories by a trap label $k$, but since the traps move independently we can omit the label. Furthermore, we go to the thermodynamic limit $K, V \rightarrow \infty$ while keeping the ratio $\rho=K / V$ fixed. This leads to the $A$ survival probability

$$
P(t)=E^{(a)}\left\{e^{-\rho E^{(b)}\left\{W\left[\boldsymbol{a}_{t}-\boldsymbol{b}_{t}\right]\right\}}\right\}
$$

where $W\left[\boldsymbol{a}_{t}-\boldsymbol{b}_{t}\right]$ is the functional of the trajectories $\boldsymbol{a}_{t}$ and $\boldsymbol{b}_{t}$

$$
W\left[\boldsymbol{a}_{t}-\boldsymbol{b}_{t}\right]=\int_{R^{d}} d \boldsymbol{B}_{0}\left(1-e^{-\int_{0}^{t} v\left(\boldsymbol{a}_{\tau}-\boldsymbol{b}_{\tau}-\boldsymbol{B}_{0}\right) d \tau}\right) .
$$

The exact problem has thus been reduced to an effective two-body problem involving a single $A$ particle and a single $B$ trap. Nevertheless, it unfortunately does not seem possible to evaluate the survival probability exactly from this expression, the main mathematical difficulty being that the average over all possible trajectories $\boldsymbol{b}_{t}$ has to be performed for each fixed $\boldsymbol{a}_{t}$. Only after this average has been performed can one then go on to carry out the further average over the $A$ particle trajectories. This appears to be a non-tractable mathematical problem, and recourse has to be made to controllable approximations. We do it here by constructing lower and upper bounds on $P(t)$ and identifying conditions and regimes where these converge asymptotically.

\section{LOWER BOUND}

A lower bound was originally devised in [18] for diffusive particles and traps and was extended in [7, 8, 10] to the case of (sub)diffusive particles and traps. We adapt this method to the current situation in which the traps $B$ perform continuous-time random walks, so that we may restate this bound in the language introduced above. Following Bray and Blythe, we introduce a notional spherical volume $V_{l}$ of radius $l$ centered at the origin, and pick only those realizations of initial trap distributions for which this volume is completely devoid of traps. The probability that the region will initially be empty of traps is

$$
P_{\text {void }}(l) \sim \exp \left(-v_{d} \rho l^{d}\right) .
$$

We furthermore introduce the probability $P_{A}(l ; t)$ that the $A$ particle does not leave the notional volume $V_{l}$ during time $t[18]$,

$$
P_{A}(l ; t) \sim \exp \left(-z_{d}^{2} D_{A} t /(l-a)^{2}\right) .
$$


Thirdly, we introduce the probability that no $B$ particle enters the notional volume up to time $t$, that is, the probability that an immobile $d$-dimensional target of radius $l$ survives up to time $t$ in the presence of a concentration $\rho$ of traps all of which perform subdiffusive motion [9]:

$$
P_{B}(l ; t) \sim \begin{cases}\exp \left(-\rho \frac{\left(4 \pi D_{B} t^{\gamma}\right)^{d / 2}}{\Gamma(1-d / 2) \Gamma(1+\gamma d / 2)}\right), & d<2 \\ \exp \left(-\rho \frac{4 \pi D_{B} t^{\gamma}}{\Gamma(1+\gamma) \ln \left(4 D_{B} t^{\gamma} / l^{2}\right)}\right), & d=2 \\ \exp \left(-\rho \frac{2(d-2) \pi^{d / 2} l^{d-2} D_{B} t^{\gamma}}{\Gamma(d / 2) \Gamma(1+\gamma)}\right), & d>2 .\end{cases}
$$

Here $\Gamma$ is the gamma function, and $D_{B}$ is the anomalous diffusion coefficient of the traps, that is, the coefficient in the mean square displacement relation $\left\langle r^{2}\right\rangle=2 d D_{B} t^{\gamma} / \Gamma(1+\gamma)$.

Since the functional in Eq. (9) is positive definite, the two latter constraints on the trajectories of $A$ and the $B$ s naturally lead to a lower bound on the survival probability $P(t)$. Furthermore, for these constrained trajectories the functional in Eq. (11) is strictly equal to zero, and hence Eq. (9) restricted in this way is equal to unity. As a consequence, the probabilities associated with the random processes $\boldsymbol{a}_{t}$ and $\boldsymbol{b}_{t}$ subject to these constraints can simply be factored, immediately leading to the lower bound on $P(t)$

$$
P(t) \geq P_{\text {void }}(l) P_{A}(l ; t) P_{B}(l ; t) \equiv P_{L}(l ; t) .
$$

Finally, we note that this lower bound $P_{L}(l ; t)$ is in fact a family of lower bounds dependent on the radius $l$ of the notional volume separating particle and traps. This radius can be chosen to give the best lower bound, that is, the maximal lower bound, which we simply denote as $P_{L}(t)$. The optimal radii are shown explicitly in Appendix $\mathrm{A}$, and are shown to depend on dimensionality and on $\gamma$. The associated optimal lower bounds for the survival probability of $A$, which also depend on dimensionality and on $\gamma$, then immediately follow. We thus have

$$
P(t) \geq P_{L}(t)
$$

where, for $d<2$,

$$
P_{L}(t) \sim \begin{cases}\exp \left(-k(d) \rho^{2 /(d+2)}\left(D_{A} t\right)^{d /(d+2)}\right), & \gamma<\frac{2}{d+2} \\ \exp \left(-\frac{\rho\left(4 \pi D_{B} t^{\gamma}\right)^{d / 2}}{\Gamma(1-d / 2) \Gamma(1+\gamma d / 2)}\right), & \gamma>\frac{2}{d+2}\end{cases}
$$

For $d=2$ we have

$$
P_{L}(t) \sim \begin{cases}\exp \left(-\left(4 \pi z_{2}^{2} \rho D_{A} t\right)^{1 / 2}\right), & \gamma<\frac{1}{2} \\ \exp \left(-\frac{8 \pi \rho D_{B} t^{\gamma}}{(2 \gamma-1) \Gamma(1+\gamma) \ln t}\right), & \gamma>\frac{1}{2}\end{cases}
$$

For $d>2$ the results are

$$
P_{L}(t) \sim \begin{cases}\exp \left(-k(d) \rho^{2 /(d+2)}\left(D_{A} t\right)^{d /(d+2)}\right), & \gamma<\frac{2}{d+2} \\ \exp \left(-d\left(\frac{(d-2) \pi^{d / 2} \rho D_{B} t^{\gamma}}{\Gamma(d / 2) \Gamma(1+\gamma)}\right)^{2 / d}\left(\frac{z_{d}^{2} D_{A} t}{d-2}\right)^{(d-2) / d}\right), & \gamma>\frac{2}{d+2}\end{cases}
$$

There is thus a change in behavior of the lower bound in all dimensions when $\gamma$ crosses the value $2 /(d+2)$.

\section{UPPER BOUNDS}

\section{A. Pascal Principle}

An upper bound on the survival probability $P(t)$ of the diffusive particle was recently derived on the basis of the so-called "Pascal Principle," which states that the $A$ particle survives longer if it remains still than if it moves. The problem with a static $A$ particle and moving traps is the so-called "target problem," and consequently we label this upper bound on the survival probability as $P_{U \text {,target }}(t)$. The Pascal Principle was conjectured in [18] and was proved in [20] for $d<2$ for conventional diffusive motion. In [21] the statement was proved for a rather general class of random walks on $d$-dimensional lattices. A 
similar statement was introduced more than a decade earlier in [22] in the context of excitation energy migration. This upper bound is given by Eq. (14) obtained in [9] if we set $l=a$, the radius of the $A$ particle. For visual ease we explicitly rewrite Eq. (14) with this replacement,

$$
P_{U, \text { target }}(t) \sim \begin{cases}\exp \left(-\rho \frac{\left(4 \pi D_{B} t^{\gamma}\right)^{d / 2}}{\Gamma(1-d / 2) \Gamma(1+\gamma d / 2)}\right), & d<2 \\ \exp \left(-\rho \frac{4 \pi D_{B} t^{\gamma}}{\Gamma(1+\gamma) \ln \left(4 D_{B} t^{\gamma} / a^{2}\right)}\right), & d=2 \\ \exp \left(-\rho \frac{2 \pi^{d / 2} a^{d-2} D_{B} t^{\gamma}}{\Gamma(d / 2) \Gamma(1+\gamma)}\right), & d>2 .\end{cases}
$$

To clearly tie together the various notations introduced so far, we note that

$$
P_{B}(a ; t) \equiv P^{A}(t) \equiv P_{U, \text { target }}(t)
$$

and

$$
P(t) \leq P_{U, \text { target }}(t)
$$

However, this upper bound when associated with the lower bound is not always sufficiently tight to provide the desired information about the asymptotic survival probability of the diffusive particle. We thus introduce and alternative new upper bound, which in some cases is lower than the above. In the next section we then explicitly pick the bounds to be used and exhibit the information that can be obtained from them.

\section{B. Anti-Pascal Principle}

This new upper bound is based on what we will call the "Anti-Pascal Principle." We will show that the worst possible strategy for traps in their search for a target is to remain immobile. Random motion, even uncorrelated with the motion of the target, enhances the probability to encounter the target. In other words, the diffusing particle $A$ survives longer if the traps remain still. The problem with a moving $A$ particle in a sea of static traps is the so-called "trapping problem," and so we label this upper bound on the survival probability as $P_{U, \text { trapping. }}$. Again, to clearly tie together various notations we note that $P^{B}(t) \equiv P_{U, \text { trapping }}(t)$, and the upper bound just introduced then says that

$$
P(t) \leq P_{U, \text { trapping }}(t)=P^{B}(t)=E^{(a)}\left\{e^{-\rho W\left[\boldsymbol{a}_{t}\right]}\right\} .
$$

To prove Eq. (23) we make use of Jensen's inequality for convex functions, which for our model can be stated as

$$
\left.e^{-\rho E^{(b)}\left\{W\left[\boldsymbol{a}_{t}-\boldsymbol{b}_{t}\right]\right.}\right\} \leq E^{(b)}\left\{e^{-\rho W\left[\boldsymbol{a}_{t}-\boldsymbol{b}_{t}\right]}\right\}
$$

(Note that this inequality is generally used to derive a lower bound. Indeed, when applied to the $E^{(a)}$ average in Eq. (10) it yields a lower bound which is exactly the Smoluchowski-type result [17].) Consequently, we have the following upper bound:

$$
P(t) \leq E^{(a)}\left\{E^{(b)}\left\{e^{-\rho W\left[\boldsymbol{a}_{t}-\boldsymbol{b}_{t}\right]}\right\}\right\} \equiv E^{(c)}\left\{e^{-\rho W\left[\boldsymbol{c}_{t}\right]}\right\},
$$

where $E^{(c)}\{\ldots\}$ denotes an average with respect to the trajectories $\boldsymbol{c}_{t}=\boldsymbol{a}_{t}-\boldsymbol{b}_{t}$ of a "fictitious" particle $C$ of radius $a$ which starts its motion at $\boldsymbol{B}_{0}$.

Note that $W\left[\boldsymbol{c}_{t}\right]$ has a clear geometric interpretaion - it defines the volume swept by the fictitious particle $C$ within the time interval $(0, t)$ and is thus an analog of the so-called Wiener sausage for conventional diffusive motion. Clearly, $W\left[\boldsymbol{c}_{t}\right]$ is a non decreasing function of time $t$ or, more precisely, of the number $N$ of jumps made by the fictitious particle within the time interval $(0, t)$. This number has two contributions, $N=N_{A}+N_{B}$, where $N_{A}$ is the number of jumps made by particle $A$ and $N_{B}$ the number of jumps made by a trap. Setting $N_{B}=0$, i.e., supposing that the trap is immobile, clearly diminishes the total number of jumps and hence diminishes $W\left[\boldsymbol{c}_{t}\right]$. In this way we tighten the bound in Eq.(25) to arrive at the desired inequality (23) We have thus established the second upper bound (23) for the survival probability of $A$. The maximal information about the survival probability is thus obtained from the lower bounds presented in Eqs. (16)-(19) and the smaller of the upper bounds (22) with (20) and (23) with (2). 


\section{COLLECTING RESULTS AND BOUNDING THE SURVIVAL PROBABILITY}

In this section we collect our detailed results, exhibited by displaying values for the constants $z$ and $\theta$ in Eq. (6) when the upper and lower bounds converge asymptotically, and providing bounds for the exponent $z$ when they do not. The results for the exponent $z$ as a function of the subdiffusive trap exponent $\gamma$ are shown for integer dimensions $d=1,2$, and 3 in Fig. 1 Even before displaying the explicit results we note the following:

- When $\gamma<2 /(d+2)$ the asymptotic survival probability of the diffusive $A$ particle in the sea of subdiffusive traps is the same as it is for immobile traps. The question asked in the introduction, whether trapping tails associated with immobile traps withstand sufficiently slow sub-diffusion, is thus answered in the affirmative, with a precise dimension-dependent characterization of what is "sufficiently slow." This behavior, obtained by the asymptotic convergence of the bounds $P_{L}(t)$ and $P_{U, \text { trapping }}(t)$, is shown for the exponent $z$ by the thick solid lines with superimposed circles in Fig. 1 In this regime the survival probability is thus of the Donsker-Varadhan form.

- When $\gamma$ crosses the value $2 /(d+2)$ there is a kind of dynamical phase transition. For $d \leq 2$ the survival probability of the diffusive particle in the sea of now more rapidly moving subdiffusive traps decays as it would if the particle remains immobile. This is indicated by the thin solid lines with superimposed circles in the first two panels of Fig. 11 and results from the asymptotic convergence of the bounds $P_{L}(t)$ and $P_{U, \text { target }}(t)$. In this regime the survival probability is thus of the Bramson-Lebowitz form generalized to subdiffusive traps.

- For $d>2$ and $\gamma>2 /(d+2)$ the situation is left somewhat uncertain: we are only able to bound the decay exponent but not determine it uniquely, because neither upper bound converges asymptotically to the lower bound. All we can say is that the survival probability decay exponent $z$ lies in the triangular region bounded by the thin solid line with superimposed circles (associated with the lower bound), the thick solid line (associated with $P_{U, \text { trapping }}$ ), and the thin solid line (associated with $\left.P_{U, \text { target }}\right)$, as indicated in the figure caption.

Finally, we collect the explicit results described above in Table $\nabla$ The reported results are for the exponents $z$ and the prefactors $\theta$ in Eq. (6). Again, we note that the results for $z$ are sketched explicitly for $d=1,2,3$ in Fig. 1

\section{CONCLUSIONS}

In conclusion, we have rigorously determined the survival probability of a particle diffusing in a $d$-dimensional medium in the presence of a concentration of traps performing subdiffusive random motion. We have arrived at our results by calculating a lower bound and two alternative upper bounds for the survival probability. One of the upper bounds, based on the "Pascal Principle," is obtained by assuming the particle to remain immobile. The other, based on the "Anti-Pascal Principle," is found by assuming that the traps remain immobile. We can then choose the tighter (lower) upper bound. Results for the asymptotic survival probability of the particle can thus be extracted if the lower bound and one of the upper bounds converge asymptotically.
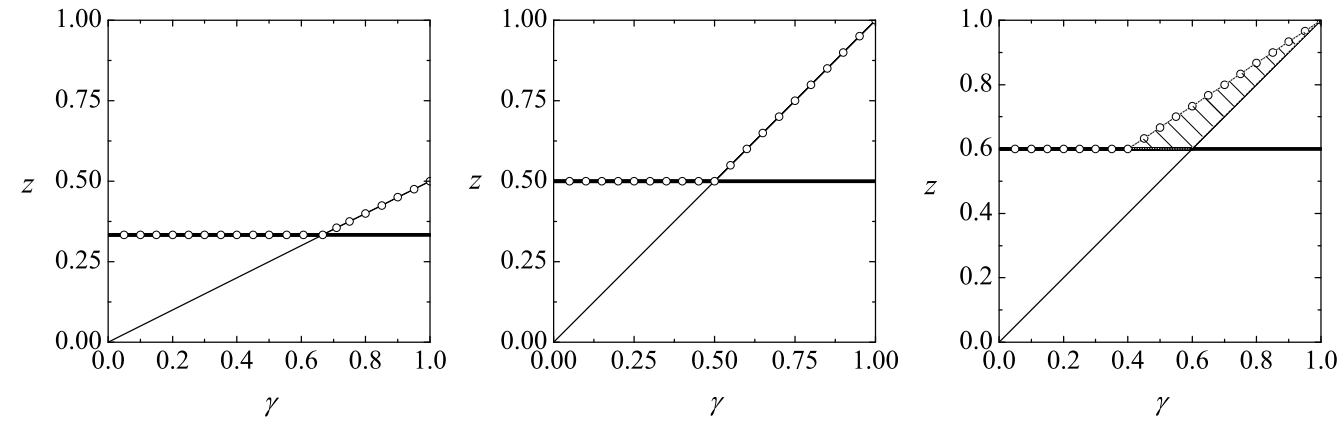

FIG. 1: Asymptotic results for the survival probability of a diffusive particle in a sea of subdiffusive traps. Plotted is the exponent $z$ as expressed in Eq. 6), or bounds on this exponent, as a function of the trap subdiffusion exponent $\gamma$. First panel: $d=1$; second panel: $d=2$. Third panel: $d=3$. The solid lines are upper bounds; specifically, the thick solid lines are Pascal upper bounds (immobile particle) while the thin solid lines are Anti-Pascal upper bounds (immobile traps). The solid lines with superimposed circles are lower bounds. Thus the first and second panels show that for $d \leq 2$ the Pascal (Donsker-Varadhan) and lower bound lines coincide for $\gamma \leq 2 /(d+2)$. The behavior changes when $\gamma$ crosses the value $2 /(d+2)$, leading to a coincidence of the Anti-Pascal (Bramson-Lebowitz) and lower bound lines. For $d \leq 2$ the asymptotic exponent is thus obtained for all values of $\gamma$. The third panel for $d=3$ shows convergence of the upper (Pascal) and lower bounds for $\gamma \leq 2 /(d+2)$. However, when $\gamma>2 /(d+2)=2 / 5$ the situation is uncertain. The exponent $z$ now lies in the triangular region delimited by the lower bound and the Pascal $(2 /(d+2) \leq \gamma \leq d /(d+2))$ or Anti-Pascal $(d /(d+2) \leq \gamma \leq 1)$ upper bound. 


\begin{tabular}{|c|c|c|c|c|}
\hline Dimension $d$ & $\begin{array}{c}\text { Trap Subdiffusive } \\
\text { Exponent } \gamma\end{array}$ & $\begin{array}{c}\text { Optimal } \\
\text { Upper Bound }\end{array}$ & $\begin{array}{l}\text { Survival Probability } \\
\text { Exponent } z\end{array}$ & $\begin{array}{c}\text { Survival Probability } \\
\text { Prefactor } \theta\end{array}$ \\
\hline $1 \leq d<2$ & $\begin{array}{l}0 \leq \gamma \leq \frac{2}{d+2} \\
\frac{2}{d+2} \leq \gamma \leq 1\end{array}$ & $\begin{array}{c}P_{U, \text { trapping }} \\
P_{U, \text { target }}\end{array}$ & $\begin{array}{l}\frac{d}{d+2} \\
\frac{\gamma d}{2}\end{array}$ & $\begin{array}{l}k(d) \rho^{2 /(d+2)} D_{A}^{d /(d+2)} \\
\frac{\rho\left(4 \pi D_{B}\right)^{d / 2}}{\Gamma(1-d) \Gamma(1+\gamma d / 2)}\end{array}$ \\
\hline$d=2$ & $\begin{array}{l}0 \leq \gamma \leq \frac{1}{2} \\
\frac{1}{2}<\gamma \leq 1\end{array}$ & $\begin{array}{c}P_{U, \text { trapping }} \\
P_{U, \text { target }}\end{array}$ & $\begin{array}{l}\frac{1}{2} \\
\gamma\end{array}$ & $\begin{array}{c}k(2)\left(\rho D_{A}\right)^{1 / 2} \\
\frac{1}{\gamma} \leq \frac{\Gamma(1+\gamma)}{4 \pi \rho D_{B}} \theta \leq \frac{2}{2 \gamma-1}\end{array}$ \\
\hline$d>2$ & $\begin{array}{c}0 \leq \gamma \leq \frac{2}{d+2} \\
\frac{2}{d+2} \leq \gamma \leq \frac{d}{d+2} \\
\frac{d}{d+2} \leq \gamma \leq 1\end{array}$ & $\begin{array}{c}P_{U, \text { trapping }} \\
P_{U, \text { target }} \\
P_{U, \text { target }}\end{array}$ & $\begin{array}{c}\frac{d}{d+2} \\
\frac{d}{d+2} \leq z \leq \frac{d-2}{d}+\frac{2 \gamma}{d} \\
\gamma \leq z \leq \frac{d-2}{d}+\frac{2 \gamma}{d}\end{array}$ & $\begin{array}{c}k(d) \rho^{2 /(d+2)} D_{A}^{d /(d+2)} \\
? \\
?\end{array}$ \\
\hline
\end{tabular}

TABLE I: Collected results for the asymptotic survival probability exponent $z$ and prefactor $\theta$.

Following this procedure, we have shown that when the dynamical exponent $\gamma$ characterizing the growth of the second moment of the displacement of the traps is less than $2 /(d+2)$, that is, if the traps move sufficiently slowly, the decay of the survival probability of the diffusing particle in any dimension is given exactly by the Donsker-Varadhan result obtained for immobile traps. When the traps move more rapidly than this, i.e., when $\gamma>2 /(d+2)$, then in dimensions $d \leq 2$ the survival probability of the particle is identical to that of a stationary $A$ particle in a sea of mobile traps. For higher dimensions, $d>2$, our results do not uniquely determine the survival probability of the particle in this $\gamma$ regime, but they do provide tighter bounds than previously known. We close by noting the well-known difficulties that may stand in the way of reaching and verifying some asymptotic results via numerical simulations (see [8] and references therein). Such simulations continue to pose an interesting challenge.

\section{Acknowledgments}

The research of S.B.Y. has been supported by the Ministerio de Educación y Ciencia (Spain) through grant No. FIS200760977 (partially financed by FEDER funds). K.L. gratefully acknowledges the support of the National Science Foundation under Grant No. PHY-0354937. The research of O.B. and G.O. is partially supported by Agence Nationale de la Recherche (ANR) under grant "DYOPTRI - Dynamique et Optimisation des Processus de Transport Intermittents".

\section{APPENDIX A: OPTIMAL NOTIONAL VOLUME}

In this appendix we explicitly show the optimal radius $l$ that leads to the maximal lower bound on the survival probability of the diffusive particle in the presence of a sea of subdiffusive point traps. Combining the expressions in Eqs. (12), (13) and (14) and differentiating their product with respect to $l$, we find that the optimal $l$ depends on dimensionality and on $\gamma$. For $d<2$ we find

$$
l \sim\left(\frac{2 z_{d}^{2} D_{A} t}{d v_{d} \rho}\right)^{1 /(d+2)} .
$$


For $d=2$ we obtain

$$
l \sim \begin{cases}\left(\frac{z_{2}^{2} D_{A} t}{\pi \rho}\right)^{1 / 4}, & \gamma<\frac{1}{2} \\ \left(\frac{z_{2}^{2} \Gamma(1+\gamma) D_{A} t}{4 \pi \rho D_{B} t^{\gamma}}\right)^{1 / 2} \ln \left(\frac{16 \pi \rho D_{B}^{2} t^{2 \gamma}}{z_{2}^{2} \Gamma(1+\gamma) D_{A} t}\right), & \gamma>\frac{1}{2}\end{cases}
$$

For $d>2$ we find

$$
l \sim \begin{cases}\left(\frac{2 z_{d}^{2} D_{A} t}{d v_{d} \rho}\right)^{1 /(d+2)}, & \gamma<\frac{2}{d+2} \\ \left(\frac{z_{d}^{2} \Gamma(d / 2) \Gamma(1+\gamma) D_{A} t^{1-\gamma}}{(d-2)^{2} \pi^{d / 2} \rho D_{B}}\right)^{1 / d}, & \gamma>\frac{2}{d+2}\end{cases}
$$

[1] I. M. Lifshitz, Sov. Phys. JETP 17, 1159 (1963); Sov. Phys. Usp. 7, 549 (1965).

[2] I. M. Lifshitz, S. A. Gredescul, and L. A. Pastur, An Introduction to the Theory of Disordered Systems (Wiley, New York, 1988).

[3] B. Ya. Balagurov and V. G. Vaks, Sov. Phys. JETP 38, 968 (1974).

[4] M. D. Donsker and S. R. S. Varadhan, Commun. Pure Appl. Math. 28, 525 (1975); 32, 721 (1979).

[5] A. M. Berezhkovskii, Yu. A. Makhnovskii, and R. A. Suris, Chem. Phys. 137, 41 (1989).

[6] A. Blumen, J. Klafter, and G. Zumofen, Phys. Rev. B 28, 6112 (1983); J. Klafter, A. Blumen, and G. Zumofen, J. Stat. Phys. 36, 561 (1984)

[7] S. B. Yuste and K. Lindenberg, Phys. Rev. E 72, 061103 (2005); S. B. Yuste, J. J. Ruiz-Lorenzo, and K. Lindenberg, Phys. Rev. E 74, 046119 (2006).

[8] J. J. Ruiz-Lorenzo, S. B. Yuste, and K. Lindenberg, J. Phys.: Condens. Matter 19, 065120 (2007).

[9] S. B. Yuste and K. Lindenberg, Phys. Rev. E 76, 051114 (2007).

[10] G. Oshanin, O. Bénichou, M. Coppey, and M. Moreau, Phys. Rev. E 66, 060101 (2002).

[11] G. Oshanin, S. Nechaev, A. M. Cazabat, and M. Moreau, Phys. Rev. E 58, 6134 (1998); S. Nechaev, G. Oshanin and A. Blumen, J. Stat. Phys. 98, 281 (2000).

[12] S. F. Burlatsky and G. S. Oshanin, Phys. Lett. A 145, 61 (1990); G. S. Oshanin, A. V. Mogutov and S. F. Burlatsky, Phys. Lett. A 149, 55 (1990); G. Oshanin, M. Moreau, and S. F. Burlatsky, Adv. Colloid Interface Sci. 49, 1 (1994).

[13] A. M. Berezhkovskii, Yu. A. Makhnovskii, and R. A. Suris, J. Stat. Phys. 57, 333 (1989); 65, 1025 (1991).

[14] J. W. Haus and K. W. Kehr, Phys. Rep. 150, 263 (1987).

[15] F. den Hollander and G. H. Weiss, in Contemporary Problems in Statistical Physics, edited by G. H. Weiss (SIAM, Philadelphia, 1994).

[16] M. Bramson and J. Lebowitz, Phys. Rev. Lett. 61, 2397 (1988); J. Stat. Phys. 62, 297 (1991).

[17] S. F. Burlatsky and A. A. Ovchinnikov, Sov. Phys. JETP 65, 908 (1987).

[18] A. J. Bray and R. A. Blythe, Phys. Rev. Lett. 89, 150601 (2002); R. A. Blythe and A. J. Bray, Phys. Rev. E 67, 041101 (2003).

[19] A. Blumen, J. Klafter and G. Zumofen, in Optical Spectroscopy of Glasses, edited by I. Zschokke (Reidel, Dordrecht, 1986).

[20] A. J. Bray, S. N. Majumdar, and R. A. Blythe, Phys. Rev. E 67, 060102(R) (2003).

[21] M. Moreau, G. Oshanin, O. Benichou, and M. Coppey, Phys. Rev. E 67, 045104(R) (2003); Phys. Rev. E 69, 046101 (2004).

[22] S. F. Burlatsky, G. S. Oshanin, and A. A. Ovchinnikov, Phys. Lett. A 139, 241 (1989). 